\title{
Life-Cycle Monitoring of CFRP using Piezoelectric Sensors Network
}

\author{
Xiao Liu ${ }^{a}$, Yishou Wang ${ }^{b}$ and Xinlin Qing ${ }^{c^{*}}$ \\ School of Aerospace Engineering, Xiamen University, Xiamen 361005, China \\ aliuxiao@stu.xmu.edu.cn; bwangys@xmu.edu.cn; cxinlinqing@xmu.edu.cn
}

\begin{abstract}
Keywords: Resin Infusion, Damage Identification, Piezoelectric Sensor Network, Structural Health Monitoring
\end{abstract}

\begin{abstract}
Vacuum Assisted Resin Infusion (VARI) process is suitable for manufacturing complex large-scale composite structures and has the potential for low cost and mass production. However, the inappropriate process parameters such as incomplete resin flow and the uneven cure occurred will lead to some defects such as dry spots and delamination. In the present work, the concept of Networked Elements for Resin Visualization and Evaluation (NERVE) with the piezoelectric lead-zirconate-titanate (PZT) sensors as the base unit was used to monitor the internal state of composite struture during its life-time. The capability of PZT sensors in the NERVE to monitor two important parameters during the manufacturing process including the flow front of resin and progress of reaction (POR), was investigated. The Lamb waves generated by PZT, propagating in the mold/composite, was used to measure the parameters. The resin flow front was analyzed using optical detection at the same time. The flow front position over time and the influence of the length of sensing path covered by resin were delivered. The effects of different resin cure state on Lamb signal attenuation and energy leakage were also obtained. The change of amplitude was integrated to get the POR curves, so the resin cure state could be also monitored. After the composite was demoulded, the network was used contiously to identify the artifical damages with the fused probability-based diagnostic imaging (PDI). Experimental results indicate that the NERVE has the ability to realize the full life-cycle health monitoring of composite structures.
\end{abstract}

\section{Introduction}

Advanced composite materials are being used on almost all modern aircraft due to their advantages of high specific strength, high specific stiffness and tailoring of mechanical properties. However, it is still difficult to precisely manufacture co-cured large-scale carbon fiber reinforced plastics (CFRP) structures and ensure their structural integrity through their life [1]. The life-cycle monitoring of CFRP includes the manufacturing process and the service stage. This paper is about the study demonstrating the sensing method based on the piezoelectric sensors network for continuously monitoring the internal state of composite structures.

As a new type of low-cost molding technology for large-scale composite materials with the advantages of excellent product performance, low porosity and good adaptability [2,3], the VARI has attracted more and more attention in the field of aviation and spaceflight. However, there are still many difficulties in the VARI process, such as the control of the resin flow, the prevention of dry spots and the delamination and residual stress that closely related to the control parameter of the process [4,5]. These flaws seriously jeopardizing the structural health cannot be easily corrected, because the trial-and-error approaches are inefficient and costly to determine suitable process parameters [6-8]. Therefore, it is necessary to carry out in-suit monitoring in the manufacturing process to adjust the process parameters in time to avoid the occurrence of 
defects. The VARI process is accompanied by a series of complex reaction processes, such as heat transfer, mass transfer, rheological reaction and polymerization reaction [9]. While the Lamb waves propagating in the homogeneous mold is suitable for the in-line VARI process monitoring, because part of energy of the Lamb wave will leak into the liquid or viscoelastic resin in the filling and curing process. Based on the propagation characteristics of Lamb waves, the signal feature changes of leaky Lamb waves can be used to track different stage of the manufacturing process [10-12]. Different from the previous works, this study embedded several PZT sensors into the composite to obtain Lamb wave signals in a pitch-catch way, which is suitable for the monitoring of large-scale composite.

In the service stage of CFRP, ultrasonic waves are still attractive because of their relatively long distance of propagation and sensitivity to discontinuities along the propagation path [13,14]. The active ultrasonic Lamb waves-based structural health monitoring (SHM) is considered to be one of the promising methods, and PZTs are one kind of preferred actuators and sensors [15]. Among the algorithms for diagnostic imaging, the probability-based diagnostic imaging (PDI) can weaken or even eliminate the direct interpretation of the ultrasonic guided wave signals and the effect of dispersion and anisotropy [16-18].

The Networked Elements for Resin Visualization and Evaluation (NERVE) [19] based on the concept of distributed multifunctional sensor network of SMART (Stanford Multi-ActuatorReceiver Transduction) Layer [20] can monitor the manufacturing parameters (resin flow front and cure state) in the Liquid Composites Molding (LCM) process effectively. This paper firstly carried out the in-suit monitoring of VARI process experiment to validate the influence of the length of sensing path covered by resin and different cure state on Lamb signal attenuation and energy leakage. Then after the composite was demoulded, damage identification experiment was conducted with the fusion PDI approach of multiple frequencies. The experimental results indicated that the NERVE has the ability to realize the full life-cycle health monitoring of composite structures.

\section{Manufacturing monitoring Experimental setup for VARI process}

Before the experiment, three SMART layers were hollowed out for the resin to flow easily and nine circular PZT sensors $(\Phi 8 \mathrm{~mm} \times 0.33 \mathrm{~mm})$ with the piezoelectric strain constant of about $d_{33}=510 \times 10^{-12} \mathrm{C} / \mathrm{N}$ were mounted on the bonding pad of the polyimide film Kapton ${ }^{\circledR}$ substrate, as shown in Fig. 1.

The fiber prefabrication $(400 \mathrm{~mm} \times 400 \mathrm{~mm} \times 2 \mathrm{~mm})$ consisted of eight $\mathrm{T} 300$ woven carbon fiber plies was placed in the central area of the surface of a 6061-Aluminum alloy plate mold $(600 \mathrm{~mm} \times 600 \mathrm{~mm} \times 2 \mathrm{~mm})$. Then the layers were inserted into a specific position between the sixth and seventh plies to form a sensor network which were shown in Fig. 5. After that, seal the system and pump the air. Then the ScanGenie II developed by Acellent Technologies, Inc. at Sunnyvale, USA was used for generating and receiving Lamb wave signals as the baselines of different sensing paths. Due to the slight difference in the performance of piezoelectric sensors, the optimal excitation frequencies of different paths are obtained. The sensing paths and different excitation characteristics for manufacturing monitoring in Fig. 1 are listed in Table 1. 




Fig. 1 The scene picture of the experiment for the VARI process.

Table 1 Paths and excitation characteristics of piezoelectric sensor network

\begin{tabular}{ccc|ccc|ccc}
\hline Path & Amp $(\mathrm{V})$ & $f(\mathrm{kHz})$ & Path & Amp $(\mathrm{V})$ & $f(\mathrm{kHz})$ & Path & Amp $(\mathrm{V})$ & $f(\mathrm{kHz})$ \\
\hline P4-3 & 60 & 190 & P4-2 & 60 & 180 & P8-6 & 75 & 170 \\
P5-3 & 60 & 180 & P5-2 & 70 & 190 & P9-4 & 70 & 180 \\
P6-3 & 70 & 220 & P6-2 & 70 & 180 & P9-5 & 70 & 180 \\
P7-3 & 75 & 170 & P7-4 & 70 & 190 & P9-6 & 70 & 190 \\
P8-3 & 75 & 160 & P7-5 & 60 & 180 & P7-2 & 75 & 180 \\
P9-3 & 75 & 180 & P7-6 & 60 & 190 & P8-2 & 75 & 160 \\
P4-6 & 70 & 170 & P8-4 & 70 & 160 & P9-2 & 75 & 160 \\
P7-9 & 70 & 190 & P8-5 & 75 & 160 & & & \\
\hline
\end{tabular}

Note: P4-3 means the sensing path that Sensor 4 is the actuator and Sensor 3 is the receiver in this paper.

The resin injection was carri out at room temperature. The resin used in this experiment was a mixture of epoxy resin (EC-TDS-IN2-Infusion-Resin) and curing agent (Formulated Amine) with a mixed ratio of 0.3 by weight. The low viscosity of $550 \mathrm{mPa}$.s at room temperature ensured the infusion of the production. With the injection of resin, the response signals excited by the narrowband five-peak sine waves modulated by Hanning window were collected continuously about every 2 minutes. A camera was placed at the top of the oven to track the resin flow front. After the resin filling process, clamped the resin tube and keep pumping the air. According the resin cure curves, the whole system was cured at a constant temperature $\left(50{ }^{\circ} \mathrm{C}\right)$ for 6 hours to eliminate the effect of temperature changes on the Lamb waves signal. The response signals were collected every 5 minutes in the resin curing process.

\section{Experimental results of VARI process}

For each sensing path listed in Table. 1, ninety-five sets of response signals were collected in about 6.5 hours. Among them, the first twenty sets of response signals were collected in the filling process and the others were in the curing process. The complexity of the waveguides and 
the influence of experimental environment made it very difficult to determine the modes, so the first wave packet (the fastest mode) was selected to compare the amplitude and attenuation during the whole process.

Resin filling stage

In the resin filling stage, the normalized amplitude curves versus filling times are shown in Fig. 2. Some interesting issues can be found: the amplitude fluctuated slightly before the corresponding sensing paths were covered by liquid resin. In the optical detection shown in Fig.2, the sequences of the sensors covered by resin were sensor 3,2,6,5. The order of the amplitude curves tended to decline is the same and implied the sequence of the sensing path started to be covered. Then the amplitude continued to decline because partial energy of Lamb waves would leak into the composite through the liquid-solid boundary. When the sensing paths were fully covered, the signals tended to be stable and the amplitude remained zero.
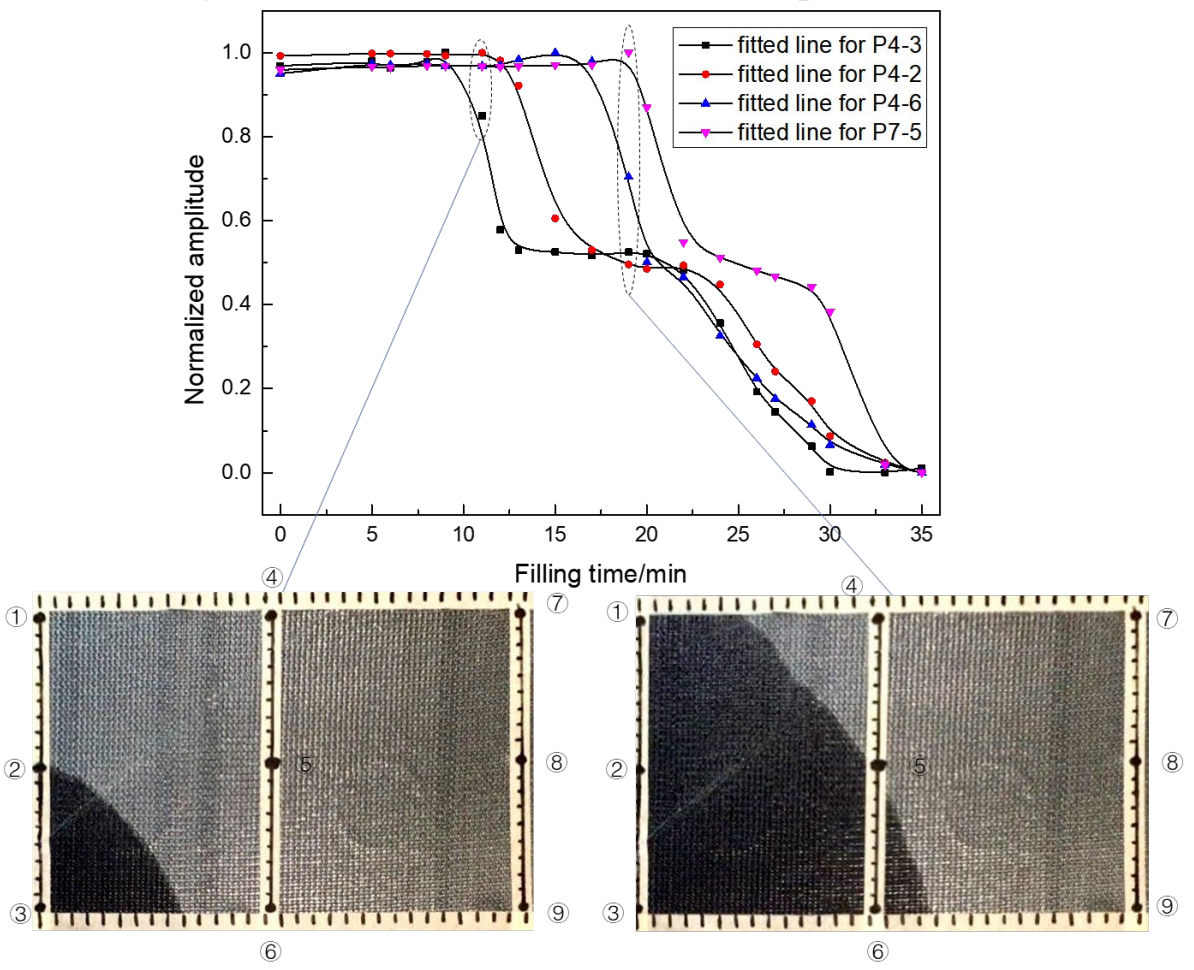

Fig. 2 The normalized amplitude curves versus filling time and the optical detection results.

In order to reveal the relationship between the resin covering length and the amplitude decrease, four long paths were chosen and the normalized amplitude curves versus length covered by resin were showed in Fig.3. It can be found that the amplitude decreased with the length covered by resin. The signal amplitudes of corresponding paths decrease sharply when the resin flow front reaches near the PZTs (less than $10 \mathrm{~cm}$ ), no matter the actuator or the receiver. However, when the flow front reached the middle of the path and was more than $10 \mathrm{~cm}$ away from the actuator and the receive, the effect of the resin covering length on the signal amplitude is much lower. Thus, the resin flow front in the filling process can be monitored by the Lamb waves effectively. 


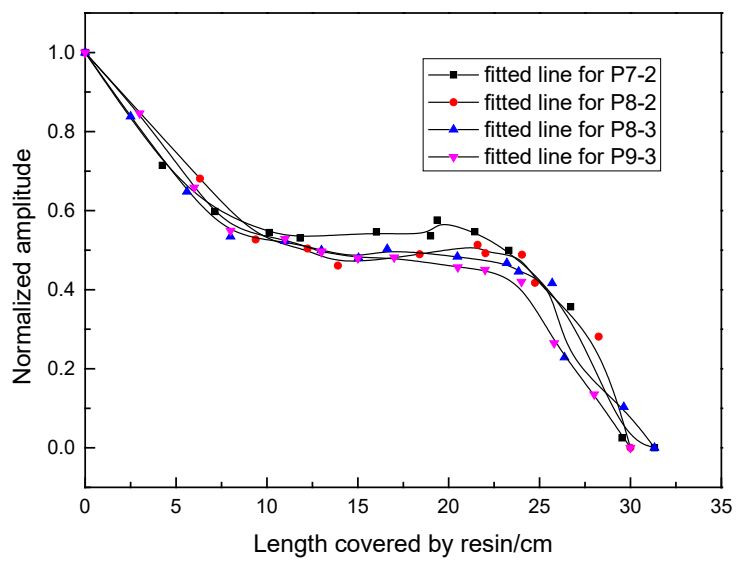

Fig. 3 The relationship between the resin covering length and the amplitude.

\section{Resin curing stage}

In the curing stage, the thermoset resin undergoes a molecular crosslinking process during which it changes irreversibly from being viscous liquid to rubbery state and finally to rigid and highly cross-linked polymer solids. The amplitudes rise sharply with the crosslinking reaction and fluctuate slightly until they stabilize.

When the resin is fully cured, it is in the glass state and can be considered as an elastic material. The effect of viscoelasticity on guided waves is negligible. So, the attenuation of the Lamb waves $\alpha$ at cure time $t$ was calculated from Eq. (1):

$$
\alpha=20 \log \left(A_{0} / A_{t}\right) / L
$$

where $A_{t}$ and $A_{0}$ are the amplitudes of the first peak in the waveform obtained at cure time $t$ and the end of the process, $L$ is the length of the sensing path.

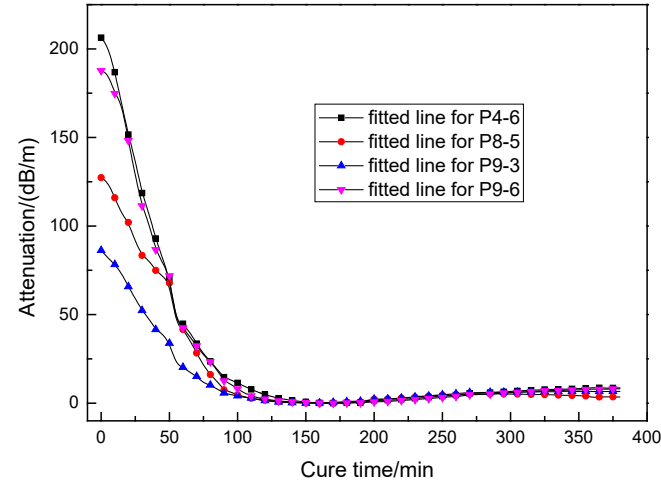

(a)

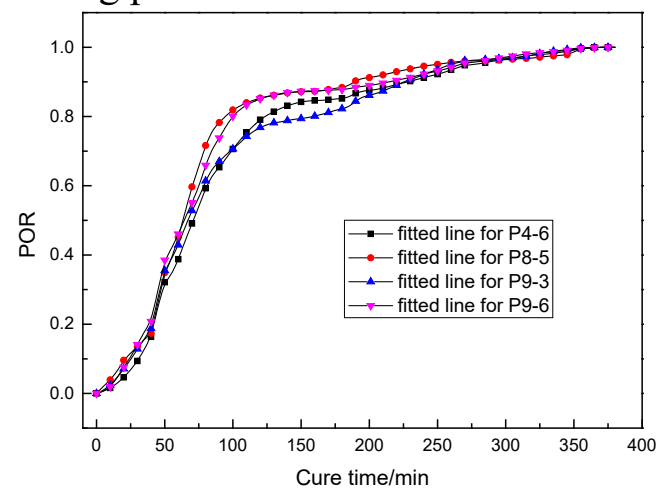

(b)

Fig. 4 (a)The attenuation of the Lamb waves obtained during the cure process; (b) The progress of crosslinking reaction over cure time.

The attenuation curves of the Lamb waves obtained during the cure process are shown in Fig. 4(a). The attenuations reach the maximum at the beginning and decrease with the cure process. At 150 minutes after cure, the attenuations become close to zero and the rate of reaction is very slow until the resin is fully cured.

The normalized amplitude represents the cross-linking process, and the changes of amplitude represent the changes in the rate of reaction. Since the cross-link reaction is irreversible, so the absolute values of changes are integrated using Eq. (2): 


$$
\int_{t_{1}}^{t_{n}} X d t \approx \sum_{i=1}^{n-2}\left(t_{i+1}-t_{i}\right) \frac{1}{2}\left(\left|x_{i+2}-x_{i+1}\right|+\left|x_{i+1}-x_{i}\right|\right)
$$

in which $x_{i}$ is the amplitude at time $t_{i}$. Then the data is normalized to get the POR curves of different sensing paths over cure time, which are shown in Fig. 4(b).It can also be concluded that the progress of crosslinking reaction increases with the cure time, and becomes slow after 150 minutes and the resin is fully cured after 300 minutes. So the real-time monitoring of resin cure process can be achieved by the POR curves using the NERVE.

\section{Service monitoring} Experimental setup for damage identification

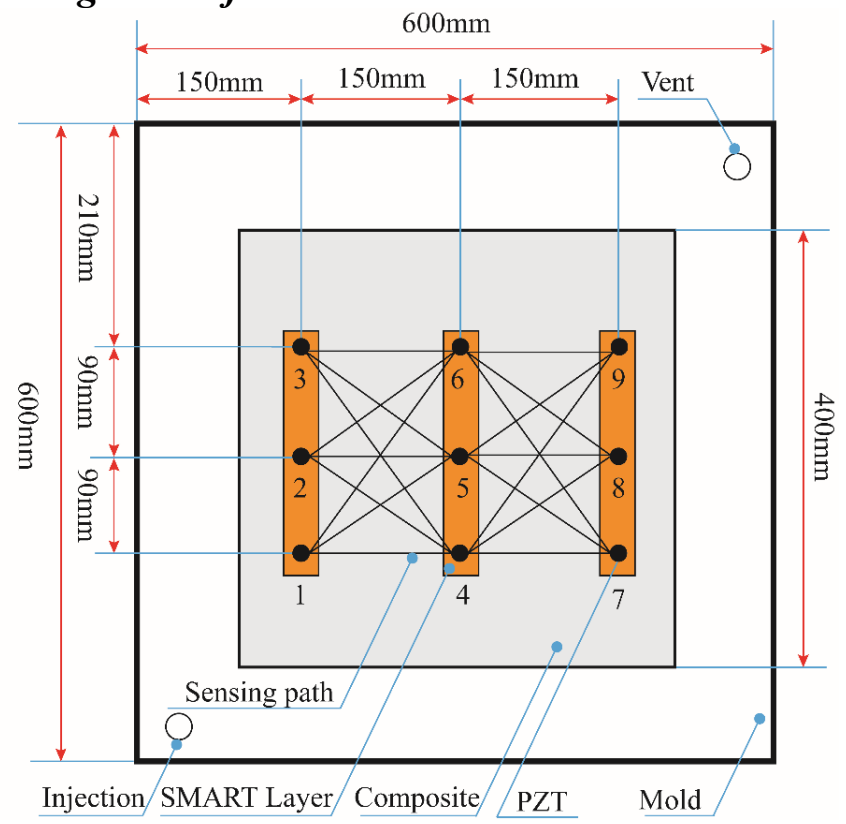

Fig. 5 The network of sensing paths for damage identification.

After the manufacturing process, the composite was demoulded and the damage identification experiment was carried in the room temperature. The network of sensing paths for damage identification is showed as Fig. 5. To reduce the cost, three cases of six artificial damages with the solid adhesive tape of $20 \mathrm{~mm}$ in the diameter and $2 \mathrm{~mm}$ in the thickness were bonded on the surface of the panel in this part. Different from the manufacturing process, the frequencies that are more sensitive to the damage in the composite plate needed to be selected. Because the ultrasonic waves decay faster in CFRP than in aluminum, so lower frequencies should be selected. The excitation frequencies are $50 \mathrm{kHz}, 60 \mathrm{kHz}$ and $70 \mathrm{kHz}$ for the sensing path in Fig. 5. Three frequencies are fused and the scaling parameter $\beta$ is set to be 0.3 .

\section{Experimental results for damage identification}

According to the relationship with the sensing paths, six artificial damages can be divided into three cases: the intersection of three paths, the intersection of two paths and only on one path. The different diagnostic images concerning the presence of probability of damages are shown as Fig. 6. 




(a)

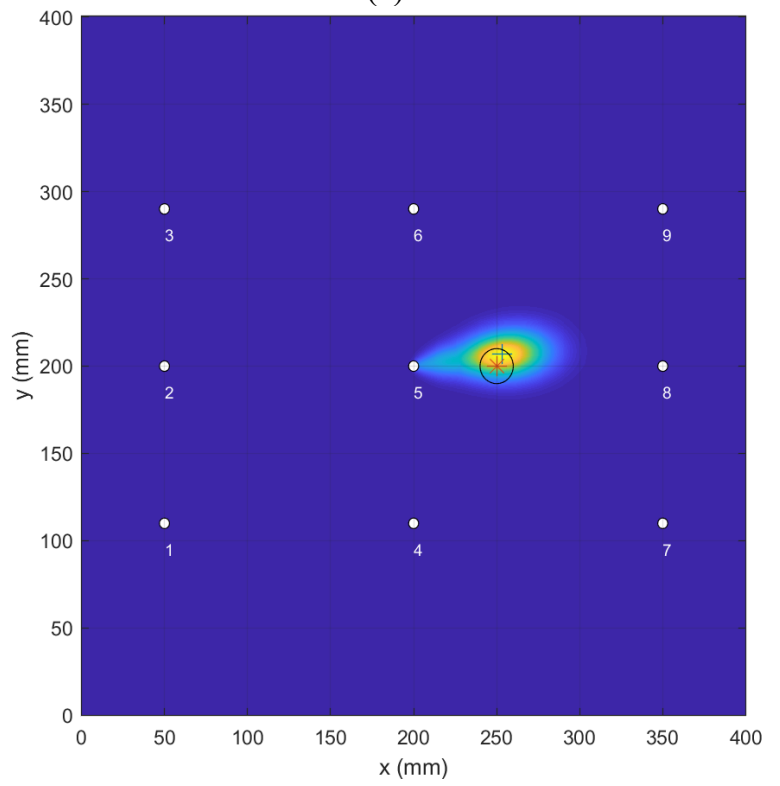

(c)

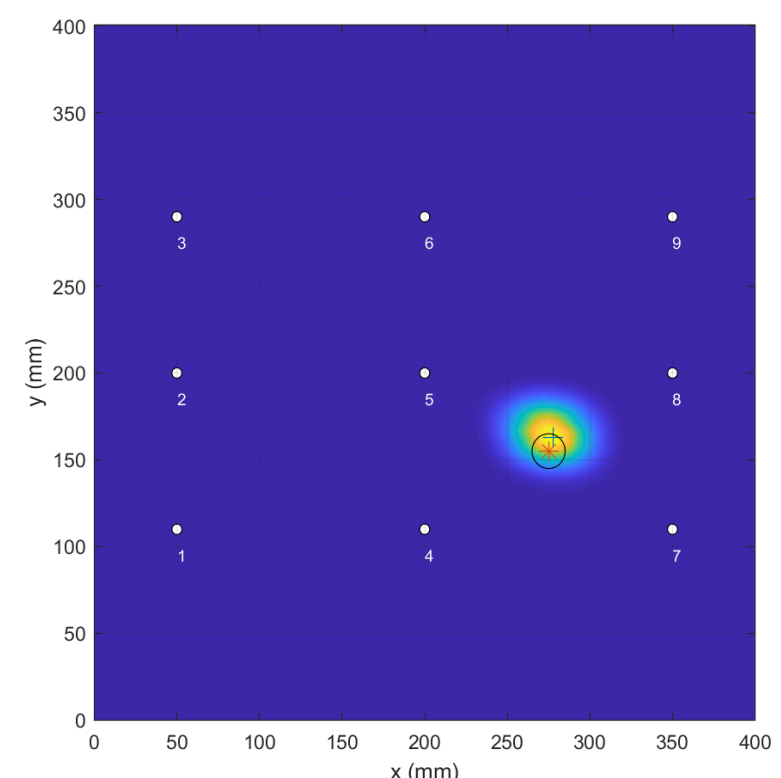

(b)

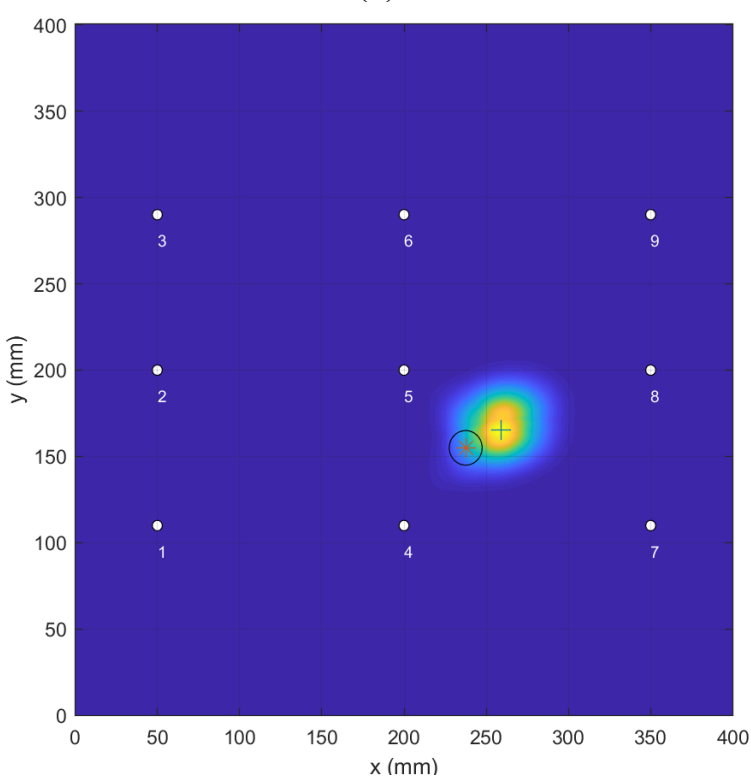

(d)

Fig. 6 The diagnostic images concerning the presence of probability of three cases of damages (the colour coding indicates its relative probability (blue: low to yellow: high), the blue "+ " is the damage centers calculated by the fused PDI and the red "*” is the actual artificial damage center). (a) intersection of three paths, (b) intersection of two paths, (c) and (d) only one path.

The Fig. 6 gives the diagnostic images and the results indicate that the fused PDI algorithm can locate different damages accurately. In order to further quantify the damage detection capability of embedded sensor network, the damage center of actual damage and the results obtained by the PDI are extracted and the relative distances between them are calculated. The relative error which is the ratio of relative distance to the length of the shortest sensing path (150 $\mathrm{mm}$ in this paper) is also calculated and showed in Table 2. The maximum localization error is $24.19 \mathrm{~mm}$ and the relative error is $16 \%$. The results further indicate that the embedded sensor 
network and the fused PDI algorithm are capable of pinpointing damage in the service stage of composite structure.

Table 2 The damage identification results of embedded sensor network using fused PDI.

\begin{tabular}{ccccc}
\hline Number & Actual damage & Fused PDI & Relative distance $(\mathrm{mm})$ & Relative error \\
\hline 1 & $(275,200)$ & $(265.5,207)$ & 11.80 & $8 \%$ \\
2 & $(275,155)$ & $(277.5,162)$ & 7.43 & $5 \%$ \\
3 & $(275,245)$ & $(279,243)$ & 4.47 & $3 \%$ \\
4 & $(100,200)$ & $(101.5,204.5)$ & 4.74 & $3 \%$ \\
5 & $(250,200)$ & $(253.5,207)$ & 7.83 & $5 \%$ \\
6 & $(237.5,155)$ & $(258.5,167)$ & 24.19 & $16 \%$ \\
\hline
\end{tabular}

\section{Conclusion}

The experimental results indicate that the NERVE has the ability to realize the full life-cycle health monitoring of composite structures, including the resin flow front and cure state in manufacturing process and the damage identification in the service stage.

In the resin filling process of the VARI experiment, partial energy of Lamb waves would leak into the composite structure through the liquid-solid boundary. The order of the amplitude curves tends to decline implied the sequence in which the sensing path starts to be covered. When the resin flow front reaches near the PZTs (less than $10 \mathrm{~cm}$ ), no matter the actuator or the receiver, the signal amplitudes of corresponding paths decrease sharply. But the effect of the resin covering length on the signal amplitude is much lower when the flow front is more than $10 \mathrm{~cm}$ away from the sensors. In the resin curing stage, the attenuations reach the maximum at the beginning and decrease with the cure process. At 150 minutes after cure, the attenuations become close to zero and the rate of reaction is very slow until the resin is fully cured, which is the same as the progress of crosslinking reaction by integrating the absolute values of amplitude changes.

After the composite is demoulded, six artificial damages with a kind of solid adhesive tape are bonded on the surface of the panel and the damages are identified using the fused PDI algorithm. The maximum localization error is $24.19 \mathrm{~mm}$ and the relative error is $16.13 \%$. The diagnostic images concerning the presence of probability of damages and the damage localization results indicate that the embedded PZT sensor network is capable of pinpointing damage in the service stage of composite structure.

By comparing the propagation characteristic of Lamb waves, the in-line monitoring of the internal state of composite structure during its life time can be achieved by the NERVE under constant temperatures. But the effect of temperature changes on Lamb wave signals should be studied and the corresponding temperature compensation strategy is still a challenging problem needed to be solved in the real manufacturing and service environment.

\section{Reference}

[1] Minakuchi S, Takeda N, Takeda S I, et al. Life cycle monitoring of large-scale CFRP VARTM structure by fiber-optic-based distributed sensing[J]. Composites Part A: Applied Science and Manufacturing, 2011, 42(6):669-676. https://doi.org/10.1016/j.compositesa.2011.02.006

[2] Laurenzi S, Casini A, Pocci D. Design and fabrication of a helicopter unitized structure using resin transfer moulding. Composites Part A: Applied Science and Manufacturing, 2014, 67:221232. https://doi.org/10.1016/j.compositesa.2014.09.007 
[3] Konstantopoulos S, Hueber C, Antoniadis I, et al. Liquid composite molding reproducibility in real-world production of fiber reinforced polymeric composites: a review of challenges and solutions. Advanced Manufacturing: Polymer \& Composites Science, 2019,5:3, 85-99. https://doi.org/10.1080/20550340.2019.1635778

[4] Park CH, Woo L. Modeling void formation and unsaturated flow in liquid composite molding processes: a survey and review. Journal of Reinforced Plastics \& Composites 2011; 30(11):957977. https://doi.org/10.1177/0731684411411338

[5] Kuentzer N, Simacek P, Advani SG, Walsh S. Correlation of void distribution to VARTM manufacturing techniques. Composites Part A: Applied Science and Manufacturing, 2007, 38(3):802-813. https://doi.org/10.1016/j.compositesa.2006.08.005

[6] Khoun L, Palardy G, Hubert P. Relation between volumetric changes of unsaturated polyester resin and surface finish quality of fiberglass/unsaturated polyester composite panels. Polymer Composites, 2011, 32(9): 1473-80. https://doi.org/10.1002/pc.21157

[7] Villière, Maxime, Guéroult, Sébastien, Sobotka V, et al. Dynamic saturation curve measurement in liquid composite molding by heat transfer analysis. Composites Part A: Applied Science and Manufacturing, 2015, 69:255-265. https://doi.org/10.1016/j.compositesa.2014.11.024

[8] Shin DD, Hahn HT. Compaction of thick composites: Simulation and experiment. Polymer Composites, 2004, 25(1): 49-59. https://doi.org/10.1002/pc.20004

[9] Wang Y, Li Y, Wu D, et al. Progress on cure monitoring for Liquid Composite Molding. Aeronautical Manufacturing Technology, 2017(19):50-59.

[10] Pavlopoulou S, Soutis C, Staszewski W J. Cure monitoring through time-frequency analysis of guided ultrasonic waves, Plastics, Rubber and Composites 41(2012)180-186. https://doi.org/10.1179/1743289811Y.0000000052

[11] Hudson T B, Grimsley B W, Yuan F G. Development of a fully automated guided wave system for in-process cure monitoring of CFRP composite laminates, Proceeding of 31st American Society for Composites Technical Conference and ASTM Committee,2016.

[12] Liu X, Li Y, Zhu J, Wang Y, Qing X. Monitoring of resin flow front and degree of cure in VARI process using multifunctional piezoelectric sensor network. Polymer Composite, accepted for publication.

[13] Predoi M V, Castaings M, Hosten B, et al. Wave propagation along transversely periodic structures[J]. Journal of the Acoustical Society of America, 2007, 121(4):1935-0. https://doi.org/10.1121/1.2534256

[14] Castaings M, Lowe M. Finite element model for waves guided along solid systems of arbitrary section coupled to infinite solid media[J]. Journal of the Acoustical Society of America, 2008, 123(2):696-0. https://doi.org/10.1121/1.2821973

[15] Qiu L, Liu M, Qing X, et al. A quantitative multidamage monitoring method for large-scale complex composite[J]. Structural Health Monitoring, 2013, 12(3):183-196.

https://doi.org/10.1177/1475921713479643 
[16] Wang D, Ye L, Lu Y, et al. Probability of the presence of damage estimated from an active sensor network in a composite panel of multiple stiffeners[J]. Composites ence \& Technology, 2009, 69(13):2054-2063. https://doi.org/10.1016/j.compscitech.2008.11.005

[17] Zhanjun Wu, Kehai Liu, Yishou Wang, et al. Validation and evaluation of damage identification using probability-based diagnostic imaging on a stiffened composite panel[J]. Journal of Intelligent Material Systems \& Structures, 2014.

[18] Liu K, Ma S, Wu Z, et al. A novel probability-based diagnostic imaging with weight compensation for damage localization using guided waves[J]. Structural Health Monitoring, 2016, 15(2):162-173. https://doi.org/10.1177/1475921715627491

[19] Qing X, Liu X, Zhu J, Wang Y. In-situ monitoring of Liquid Composite Molding process using piezoelectric sensor network[J]. Structural Health Monitoring, accepted for publication.

[20] Qing X, Beard S, Kumar K, et al. Advances in the development of built-in diagnostic system for filament wound composite structures. Composites Science \& Technology 2006; 66(11-12):1694-1702. https://doi.org/10.1016/j.compscitech.2005.11.007 